\title{
Relación entre liderazgo en seguridad y cultura en seguridad en trabajadores de tres pymes del sector industrial de Bucaramanga
}

Relationship between safety leadership and safety culture in workers of three SMEs in the industrial sector of Bucaramanga

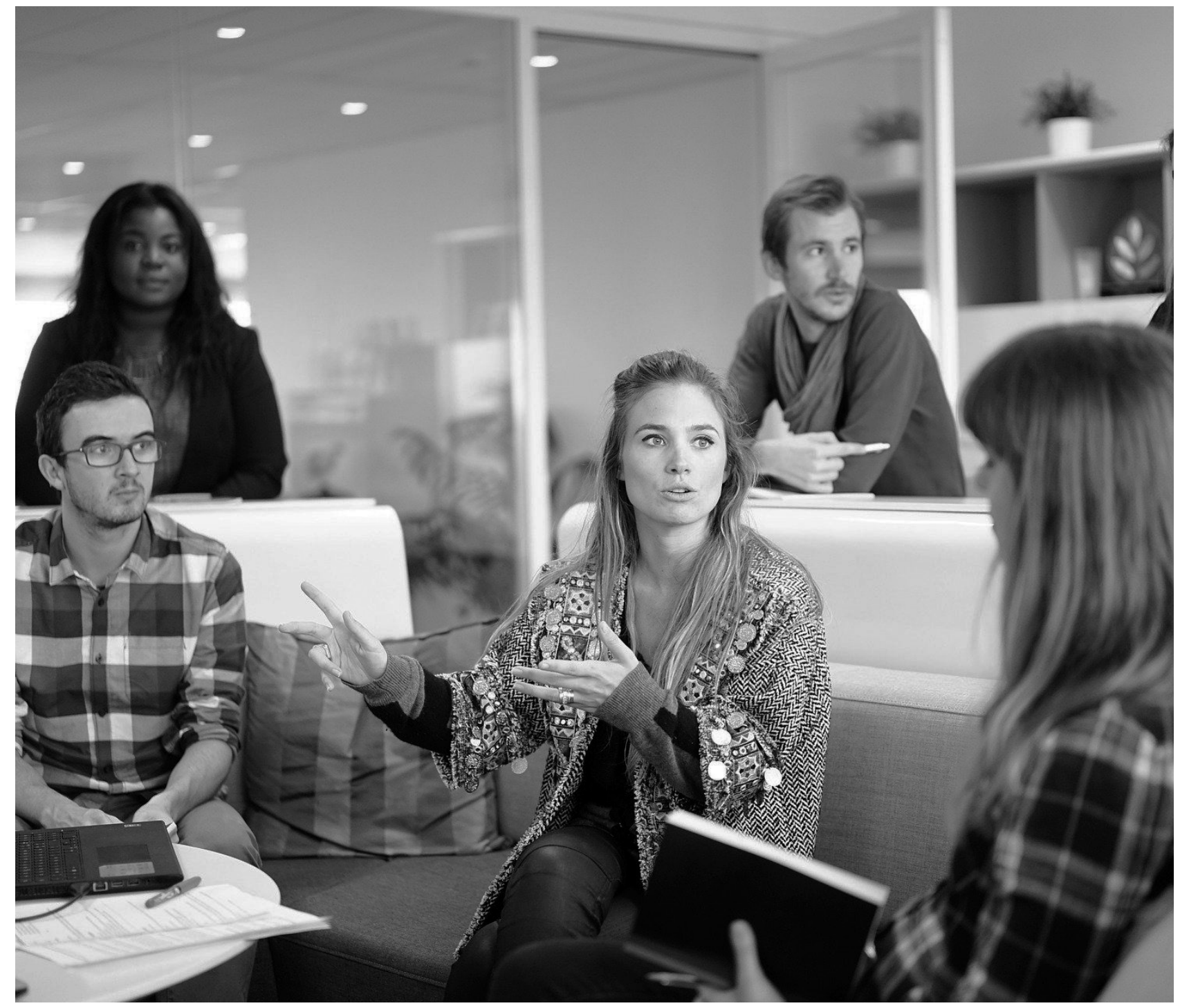




\title{
Relación entre liderazgo en seguridad y cultura en seguridad en trabajadores de tres pymes del sector industrial de Bucaramanga ${ }^{1}$ Relationship between safety leadership and safety culture in workers of three SMEs in the industrial sector of Bucaramanga
}

\author{
Germán Manuel Argüello-López², Ana Patricia Buenahora-Morales³, \\ Mónica Fernanda Mantilla-Martínez ${ }^{4}$
}

Artículo recibido en noviembre 29 de 2018; artículo aceptado en marzo 22 de 2019.

Este artículo puede compartirse bajo la Licencia Creative Commons Atribución-NoComercial-Compartirlgual 4.0 Internacional

y se referencia usando el siguiente formato: Argüello-López, G. M.; Buenahora-Morales, A. P. \& Mantilla-Martínez, M. F.

(2019). Relación entre liderazgo en seguridad y cultura en seguridad en trabajadores de tres pymes del sector industrial de

Bucaramanga. I+D Revista de Investigaciones, 14 (2), 52-63. DOI: https://doi.org/10.33304/revinv.v14n2-2019005

\begin{abstract}
Resumen
La cultura en seguridad de una organización determina el éxito en la implementación del sistema de gestión de seguridad y salud en el trabajo. Uno de los principales factores que afectan el nivel de cultura en seguridad es el liderazgo en seguridad de directivos, mandos medios y responsables del sistema de gestión. Se presentan los resultados de un estudio cuyo objetivo era determinar la relación entre "cultura en seguridad" y "liderazgo en seguridad" en empleados de tres pymes del sector industrial. Este estudio tiene un enfoque cuantitativo no experimental, de corte transversal y de alcance correlacional. Para medir las variables se utilizaron instrumentos validados en estudios previos. Se comprobó que el nivel de "cultura en seguridad" estaba determinado en gran medida por el nivel de "liderazgo en seguridad" en las tres pymes analizadas.
\end{abstract}

Palabras clave: Cultura de seguridad, clima de seguridad, pymes, industrial.

\section{Abstract}

The safety culture of an organization determines the success in the implementation of the safety and health management system at work. One of the main factors that affect the level of safety culture is the safety leadership of managers, the middle management and those in charge of the management system. This article presents the

1. Artículo de investigación, con enfoque cualitativo, resultado de un proyecto de investigación culminado, perteneciente al área de ciencias sociales, subárea salud y seguridad en el trabajo, desarrollado en el grupo de investigación Sinergia, financiado por la Universidad de Investigación y Desarrollo (UDI), Bucaramanga (Colombia). Dirección: calle 9 n. 23-55, PBX: 6352525. Fecha de inicio: enero de 2017. Fecha de terminación: diciembre de 2017.

2. Ingeniero industrial, Universidad Industrial de Santander. Magíster en Entornos Virtuales de Aprendizaje, Universidad de Panamá. Docente cátedra de la Universidad Autónoma de Bucaramanga (Colombia). Dirección: avenida 42 n. ${ }^{\circ} 48-11$, PBX: 6436111. ORCID ID: http://orcid.org/00000002-2805-6888. Correo electrónico institucional: garguello679@unab.edu.co.

3. Ingeniero industrial, UDI. Joven-investigador del grupo Sinergia. Universidad de Investigación y Desarrollo, Bucaramanga, Colombia. Dirección: calle 9 n. ${ }^{\circ} 23-55$, PBX: 6352525. ORCID ID: https://orcid.org/0000-0002-3450-9476. Correo institucional: abuenahora1@udi.edu.co.

4. Ingeniero industrial, UDI. Joven-investigador del grupo Sinergia. Universidad de Investigación y Desarrollo, Bucaramanga, Colombia. Dirección: calle 9 n. ${ }^{\circ}$ 23-55, PBX: 6352525. ORCID ID: https://orcid.org/0000-0001-6803-4731. Correo institucional: mmantilla3@udi.edu.co. 
results of a study whose objective was to determine the relationship between "safety culture" and "safety leadership" in employees of three SMEs in the industrial sector. This research study has a quantitative, non-experimental, crosssectional approach of correlational scope. To measure the variables, instruments validated in previous studies were used. It was found that the level of "safety culture" was determined to a large extent by the level of "safety leadership" in the three SMEs analyzed.

Keywords: Safety culture, safety climate, SMEs, industrial.

\section{Introducción}

Según datos de la Cámara de Comercio de Bucaramanga (2018b), el sector industrial ocupa el segundo puesto en número de empresas en el departamento de Santander. Este sector se conforma de 10.446 empresas, de las cuales $0,34 \%$ son grandes; $0,81 \%$, medianas; 3,91 \%, pequeñas; y $94,93 \%$, microempresas. Del $100 \%$ de las empresas que componen el sector industrial, el $88,72 \%$ se encuentran en el área metropolitana de Bucaramanga. Los principales subsectores que lo integran son elaboración de productos alimenticios; confección de prendas de vestir; fabricación de calzado y marroquinería; fabricación de productos elaborados de metal, excepto maquinaria y equipo; fabricación de muebles, colchones y somieres; y fabricación de joyas y artículos conexos (Cámara de Comercio de Bucaramanga, 2018a).

Según cifras de Fasecolda (2018), aún hasta diciembre de 2016 las empresas del sector industrial del área metropolitana de Bucaramanga afiliadas al sistema de riegos laborales reportaron 2257 accidentes laborales y 56 enfermedades laborales. Esto es, 1,38 accidentes laborales por empresa. Si bien este valor está por debajo del promedio nacional para el sector industrial, que está en 1,77 accidentes laborales por empresa, sigue por encima del número promedio de accidentes por empresa del área metropolitana de Bucaramanga y del ámbito nacional, con 1,06 y 1,02 , respectivamente.

Pucci (2007) propone una clasificación de las investigaciones sobre seguridad en el trabajo en tres ejes fundamentales: el individuo, el puesto de trabajo y la organización. Pucci explica que las investigaciones respecto de los individuos han girado en torno al análisis de la fiabilidad de los comportamientos partiendo de cuatro determinantes: la personalidad, la motivación, el aprendizaje y la decisión. Puyal-Español (2001) confirma esto al afirmar que en la prevención de riesgos laborales intervienen importantes aspectos psicosociales, como las actitudes, los hábitos, la percepción del riesgo y la percepción de su control, así como el nivel de satisfacción de los empleados, entre otros. Vanegas et al. (2017) aportan que en las organizaciones la gestión de riesgo debe ser planificada.
Un concepto integrador de estos elementos es el de 'cultura en seguridad', que, según la Comisión de Seguridad y Salud del Reino Unido (1993), es "el producto de valores, actitudes, competencias y pautas de comportamiento individuales y de grupo que determinan el compromiso hacia la seguridad, así como el estilo y competitividad de los programas de seguridad y salud de la organización".

De manera alternativa, Rimbau (2013) propone el concepto de 'cultura preventiva' como "el conjunto de creencias, normas, actitudes, roles, prácticas sociales y técnicas que se ocupan de minimizar la exposición de los trabajadores, directivos, clientes y público a situaciones consideradas peligrosas o nocivas".

Fernández-Muñiz et al. (2004) plantean que la cultura de seguridad, que hace parte de la cultura organizativa, está integrada por dos componentes principales: el clima de seguridad, relacionado con las actitudes y comportamientos de los trabajadores; y el sistema de gestión de la seguridad y salud laboral, entendido como el conjunto de políticas, prácticas y procedimientos relacionados con la prevención del riesgo laboral.

Al respecto, Pidgeon (1991) establece que hay tres elementos clave de la cultura preventiva: 1) existencia de normas y reglas; 2 ) actitudes positivas hacia la seguridad; y 3 ) prácticas de seguridad reflexivas.

Por otra parte, según la Agencia Ejecutiva para la Salud y la Seguridad del Reino Unido (2005), uno de los cinco factores clave que determinan la cultura de seguridad de una organización es el liderazgo, junto con la comunicación bidireccional, la participación de los empleados, la cultura de aprendizaje y la actitud hacia el castigo.

Rimbau (2013) plantea que el liderazgo en seguridad ejercido por los directivos y mandos medios se hace manifiesto, en el nivel macro, en la toma de decisiones estratégicas, y en el nivel micro, mediante los comportamientos y actitudes en el trato directo con los trabajadores. Algunos ejemplos de acciones que los directivos pueden emprender desde el nivel macro serían integrar la prevención en todas las decisiones y áreas funcionales; establecer objetivos; asignar 
responsabilidades y recursos; y favorecer la comunicación bidireccional. A nivel micro, el liderazgo en seguridad de directivos y mandos medios se demuestra en la capacidad para definir y coordinar tareas y personas, en dar ejemplo adoptando comportamientos seguros y en la calidad del trato que tienen con los subalternos.

En este sentido, el estilo de liderazgo tiene influencia en la prevención de los riesgos laborales. El liderazgo transformador orientado a la prevención es el factor que más contribuye al bienestar de los trabajadores y a la mejora del clima preventivo (Kapp, 2012). Surge entonces el concepto de'clima preventivo', conexo con los conceptos de'cultura en seguridad'y'clima organizacional', entendido como "la percepción que tienen los trabajadores de las políticas, procedimientos y prácticas relativas a la prevención". En este sentido, Neal et al. (2000) encontraron que el efecto del clima de seguridad en el desempeño de la seguridad estaba parcialmente mediado por el conocimiento y la motivación de la seguridad.

La relación entre liderazgo en seguridad y cultura en seguridad ha sido bastante estudiada. Martínez-Oropesa y Cremades (2012) encontraron relación entre cultura en seguridad y liderazgo en seguridad en empresas del sector industrial del Valle del Cauca. Por su parte, Fernández-Muñiz et al. (2004) concluyeron que el compromiso mostrado por la dirección de la empresa, a través de sus actitudes y comportamientos, condiciona de forma positiva la implicación y participación activa de los trabajadores en las actividades relacionadas con su seguridad y salud laboral.

En línea con estos hallazgos, se desarrolló el presente estudio, con el fin de medir la intensidad de la relación entre cultura en seguridad y liderazgo en seguridad, en trabajadores de tres pymes del sector industrial del área metropolitana de Bucaramanga.

\section{Metodología}

\section{Tipo de estudio}

La investigación tuvo un enfoque cuantitativo no experimental, de corte transversal y de alcance correlacional.

Con este estudio se buscaba determinar si el nivel de cultura en seguridad, entendida como los valores, actitudes, creencias y las percepciones de las personas y de las empresas en todo lo relacionado con la seguridad, era dependiente del nivel de liderazgo en seguridad de los directivos, mandos medios y responsables del sistema de gestión de seguridad y salud en el trabajo (SG-SST).

\section{Participantes}

La población meta seleccionada fueron los empleados de las pymes del sector fabricación de vidrio del área metropolitana de Bucaramanga.

Para fines del estudio, se determinó un tamaño de muestra de 82 trabajadores, considerando un nivel de confianza del $93 \%$, un error de estimación del $10 \%$, varianza desconocida y tamaño de población infinita. Los trabajadores se seleccionaron aleatoriamente de las pymes que aceptaron participar en el estudio, con respecto al número total de empleados de cada una. Los encuestados firmaron un consentimiento informado autorizando la utilización de la información recolectada para fines de la investigación.

\section{Materiales e instrumentos}

Para la medición de la variable cultura en seguridad, se utilizó el instrumento diseñado por Martínez-Oropesa y Cremades (2012), que consta de 94 ítems, de los cuales 87 están redactados en sentido positivo y 7 en sentido negativo, medibles con una escala de Likert de 5 niveles: $1=$ Nunca, $2=$ Casi nunca, $3=$ Algunas veces, $4=$ Casi siempre, $5=$ Siempre. Este instrumento mide 10 dimensiones que hacen parte de la cultura en seguridad: 1) sistemas de trabajo, procesos y prácticas de los empleados; 2) gerencia, cultura, liderazgo y prácticas gerenciales; 3) supervisión; 4) formación y desarrollo; 5) comunicación; 6) trabajo en equipo y colaboración; 7) participación de los trabajadores; 8) clima laboral y moral de los empleados; 9) conocimientos, actitudes y comportamientos; y 10) sistema de ambiente, salud y seguridad: mejora de la eficacia. El cuestionario se complementó con preguntas sobre la identificación de la empresa y del encuestado, causas y ocurrencia de accidentes laborales, conocimiento del plan de capacitaciones y capacitaciones recibidas y elementos de protección personal. Este cuestionario se puede consultar en el enlace https://goo.gl/xNWGfR

Para la medición de la variable liderazgo en seguridad, se utilizó el instrumento diseñado por Martínez-Oropesa y Cremades (2012), que consta de 31 ítems, redactados en sentido positivo, medibles con una escala de Likert de 5 niveles: $1=$ Nunca, $2=$ Casi nunca, $3=$ Algunas veces, $4=$ Casi siempre, $5=$ Siempre. El instrumento mide 3 dimensiones que hacen parte del liderazgo en seguridad: 1) liderazgo en seguridad de la alta gerencia; 2) liderazgo en seguridad del gerente (mando medio) y supervisor operacional; y 3) liderazgo en seguridad del profesional que gestiona el proceso. Este cuestionario se puede consultar en el enlace https://goo.gl/MbpwGs 
Se verificó la consistencia interna de las escalas y subescalas de ambos cuestionarios, mediante el cálculo del coeficiente alfa de Cronbach (Cervantes, 2005).

Para el análisis de los datos obtenidos de los instrumentos aplicados, se utilizó el software estadístico SPSS.

\section{Resultados}

Se entrevistaron un total de 82 trabajadores de las tres pymes participantes en el estudio, de los cuales 12 eran directivos y mandos medios, y 70, operativos (Tabla 1).

Tabla 1

Número de empleados encuestados por empresa y por nivel del cargo

\begin{tabular}{cccc}
\hline Empresa & $\begin{array}{c}\text { Directivos y } \\
\text { mandos } \\
\text { medios }\end{array}$ & Operativos & Total \\
\hline Empresa 1 & 2 & 11 & 13 \\
Empresa 2 & 2 & 11 & 13 \\
Empresa 3 & 8 & 48 & 56 \\
Total & 12 & 70 & 82 \\
\hline
\end{tabular}

Fuente: Autores.

Del $100 \%$ de los trabajadores encuestados, $82 \%$ eran hombres, y $4 \%$, mujeres.

Del $100 \%$ de los trabajadores encuestados, el $43,9 \%$ era bachiller, el 40,24 \% tenía formación técnica y el 11,39 $\%$ era profesional. Destaca que la mayor parte de los directivos y mandos medios, el 41,67\%, contaba con formación profesional, y el $42,86 \%$ de los trabajadores operativos tenía formación técnica.

Del $100 \%$ de los trabajadores encuestados, la gran mayoría, el $97,56 \%$, manifestó trabajar 48 horas semanales.

\section{Cultura en seguridad}

El nivel promedio de cultura en seguridad de los trabajadores encuestados resultó ser 4,18, con una desviación estándar de 0,596, de forma que se ubica en un nivel medio-alto (Tabla 2). A priori se observó que el nivel de cultura en seguridad es mayor en la empresa 1 con respecto a las demás.

La prueba de normalidad de Kolmogorov-Smirnov, con el ajuste de Lilliefors, llevó a descartar el comportamiento normal de la variable cultura en seguridad ( $p$-valor $=0,004$ $<0,05)$. Esto hizo necesario recurrir al uso de pruebas no paramétricas para determinar diferencias significativas por nivel de cargo y por empresa.

La prueba U de Mann-Whitney llevó a descartar la existencia de diferencia significativa entre niveles de cargo en el nivel de cultura en seguridad ( $p$-valor $=0,798>0,05$ ).

Por otra parte, el análisis de las dimensiones de la variable por nivel de cargo (Tabla 3) llevó a descartar diferencias significativas entre niveles de cargo. Esto, excepto en la dimensión trabajo en equipo y colaboración, donde se encontró que el nivel de los trabajadores operativos era significativamente mayor que el de los trabajadores del nivel directivo y mandos medios ( $p$-valor $=0,03<0,05$ ).

\section{Tabla 2}

Nivel promedio de cultura en seguridad por nivel de cargo y empresa

\begin{tabular}{ccccc}
\hline Nivel de cargo & Empresa & Media & $\begin{array}{c}\text { Desviación } \\
\text { estándar }\end{array}$ & N \\
\hline $\begin{array}{c}\text { Directivos y mandos } \\
\text { medios }\end{array}$ & Empresa 1 & 4,5000 & 0,28284 & 2 \\
& Empresa 2 & 4,1000 & 0,14142 & 2 \\
& Empresa 3 & 4,1125 & 0,39438 & 8 \\
Operativos & Total & 4,1750 & 0,36213 & 12 \\
& Empresa 1 & 4,8091 & 0,31130 & 11 \\
& Empresa 2 & 4,1091 & 0,57525 & 11 \\
& Empresa 3 & 4,0604 & 0,61842 & 48 \\
& Total & 4,1857 & 0,62954 & 70 \\
& Empresa 1 & 4,7615 & 0,31764 & 13 \\
& Empresa 2 & 4,1077 & 0,52672 & 13 \\
& Empresa 3 & 4,0679 & 0,58902 & 56 \\
& Total & 4,1841 & 0,59617 & 82 \\
\hline
\end{tabular}

Fuente: Autores.

Mediante la prueba de Kruskal-Wallis, se determinó que había diferencias significativas entre empresas en el nivel de cultura en seguridad ( $p$-valor $=1,49 \times 10-4<$ 0,05 ). Entre estas, el mayor nivel de cultura en seguridad es el de la empresa 1.

Por su parte, el análisis de las dimensiones de la variable por empresa (Tabla 4) permitió encontrar diferencias significativas entre empresas, en todas las dimensiones, excepto en la dimensión clima laboral y moral de los empleados ( $p$-valor $=0,137>0,05$ ). Salvo en esta dimensión, se observó que el nivel de las dimensiones de la variable era mayor en la empresa 1 , pues no hubo diferencias significativas entre las empresas 1 y 2 .

Por otra parte, se confirmó, mediante la prueba de 
Tabla 3

Nivel promedio por dimensiones de la cultura en seguridad por nivel de cargo

\begin{tabular}{|c|c|c|c|}
\hline Dimensión & $\begin{array}{c}\text { Tota } \\
\text { I }\end{array}$ & $\begin{array}{c}\text { Directivos y mandos } \\
\text { medios }\end{array}$ & $\begin{array}{c}\text { Operativo } \\
\text { s }\end{array}$ \\
\hline $\begin{array}{l}\text { Sistemas de trabajo, procesos y prácticas de los } \\
\text { empleados }\end{array}$ & 4,54 & 4,42 & 4,56 \\
\hline Gerencia, cultura, liderazgo y prácticas gerenciales & 4,38 & 4,33 & 4,39 \\
\hline Mandos medios (supervisores) & 3,96 & 4,12 & 3,93 \\
\hline Formación y desarrollo & 4,30 & 4,27 & 4,31 \\
\hline Comunicación & 4,01 & 4,01 & 4,01 \\
\hline Trabajo en equipo y colaboración & 4,11 & 3,75 & 4,17 \\
\hline Participación de los trabajadores & 4,24 & 4,34 & 4,22 \\
\hline Clima laboral y moral de los empleados & 4,16 & 4,13 & 4,17 \\
\hline Conocimientos, actitudes y comportamientos & 4,20 & 4,3 & 4,18 \\
\hline $\begin{array}{l}\text { Sistema de ambiente, salud y seguridad: mejora de la } \\
\text { eficacia }\end{array}$ & 4,14 & 4,2 & 4,13 \\
\hline Cultura en seguridad & 4,20 & 4,18 & 4,19 \\
\hline
\end{tabular}

Fuente: Autores.

\section{Tabla 4}

Nivel promedio por dimensiones de la cultura en seguridad por empresa

\begin{tabular}{lcccc}
\hline \multicolumn{1}{c}{ Dimensión } & Total & Empresa 1 & Empresa 2 & Empresa 3 \\
\hline Sistemas de trabajo, procesos y prácticas de los empleados. & 4,54 & 4,82 & 4,64 & 4,45 \\
Gerencia, cultura, liderazgo y prácticas gerenciales. & 4,38 & 4,74 & 4,31 & 4,32 \\
Mandos medios (supervisores) & 3,96 & 4,60 & 3,89 & 3,83 \\
Formación y desarrollo & 4,30 & 4,74 & 4,25 & 4,21 \\
Comunicación. & 4,01 & 4,72 & 3,80 & 3,90 \\
Trabajo en equipo y colaboración & 4,11 & 4,74 & 3,99 & 3,99 \\
Participación de los trabajadores. & 4,24 & 4,88 & 4,23 & 4,09 \\
Clima laboral y moral de los empleados. & 4,16 & 4,54 & 4,15 & 4,08 \\
Conocimientos, actitudes y comportamientos. & 4,20 & 4,81 & 4,02 & 4,10 \\
Sistema de ambiente, salud y seguridad: mejora de la eficacia. & 4,14 & 4,80 & 4,03 & 4,01 \\
Cultura en seguridad & $\mathbf{4 , 2 0}$ & $\mathbf{4 , 7 4}$ & $\mathbf{4 , 1 3}$ & $\mathbf{4 , 1 0}$ \\
\hline
\end{tabular}

Fuente: Autores.

Kruskal-Wallis, la existencia de diferencias significativas entre dimensiones ( $p$-valor $=3,496 \times 10-5<0,05$ ). Se utilizó el procedimiento de comparación múltiple de Dunn, para determinar que el nivel de la dimensión sistemas de trabajo, procesos y prácticas de los empleados estuviera por encima del nivel de las demás dimensiones, y que las dimensiones mandos medios, comunicación, trabajo en equipo, colaboración y sistema de ambiente, salud y seguridad estuvieran en un nivel similar y por debajo de las demás dimensiones (Tabla 5).

Mediante la prueba de Kruskal-Wallis se confirmó la existencia de diferencias significativas entre dimensiones en directivos y mandos medios ( $p$-valor $=0,0312<0,05$ ). Sin embargo, mediante el procedimiento de comparación múltiple de Dunn se descartó la existencia de diferencias significativas entre dimensiones en directivos y mandos medios (Tabla 6).

Mediante la prueba de Kruskal-Wallis, se confirmó la existencia de diferencias significativas entre dimensiones en trabajadores operativos ( $p$-valor $=6,29 \times 10-4<0,05$ ). Se verificó, mediante el procedimiento de comparación múltiple de Dunn, que el nivel de la dimensión sistemas de trabajo, procesos y prácticas de los empleados estuvo por encima de las demás dimensiones, y que las dimensiones mandos medios, comunicación y sistema de ambiente, salud y seguridad tuvieron un nivel similar, aun cuando estuvieron por debajo de las demás dimensiones (Tabla 7). 
Tabla 5

Procedimiento de comparación múltiple de Dunn: dimensiones cultura en seguridad

\begin{tabular}{lcccc}
\hline \multicolumn{1}{c}{ Dimensión } & Frecuencia & Suma de los rangos & Media de los rangos & Grupos \\
\hline Mandos medios (supervisores) & 82 & 28588,5000 & 348,6402 & $\mathrm{~A}$ \\
Comunicación & 82 & 28823,5000 & 351,5061 & $\mathrm{~A}$ \\
El trabajo en equipo y colaboración & 82 & 30823,0000 & 375,8902 & $\mathrm{~A}$ \\
$\begin{array}{l}\text { Sistema de ambiente, salud y seguridad: mejora de la } \\
\text { eficacia }\end{array}$ & 82 & 31064,0000 & 378,8293 & $\mathrm{~A}$ \\
Conocimientos, actitudes y comportamientos & 82 & 32690,5000 & 398,6646 & $\mathrm{~A}$ \\
Participación de los trabajadores & 82 & 33652,5000 & 410,3963 & $\mathrm{~B}$ \\
Clima laboral y moral de los empleados & 82 & 34047,0000 & 415,2073 & $\mathrm{~B}$ \\
Formación y desarrollo & 82 & 37022,0000 & 451,4878 & $\mathrm{~A}$ \\
Gerencia, cultura, liderazgo y prácticas gerenciales & 82 & 37614,0000 & 458,7073 & $\mathrm{~A}$ \\
Sistemas de trabajo, procesos y prácticas de los & & & $\mathrm{B}$ & $\mathrm{B}$ \\
empleados & 82 & 42285,0000 & 515,6707 & $\mathrm{~B}$ \\
\hline
\end{tabular}

Fuente: Autores.

Tabla 6

Procedimiento de comparación múltiple de Dunn: dimensiones cultura en seguridad de directivos y mandos medios

\begin{tabular}{|c|c|c|c|c|}
\hline Dimensión & Frecuencia & Suma de los rangos & Media de los rangos & Grupos \\
\hline Trabajo en equipo y colaboración & 12 & 408,0000 & 34,0000 & A \\
\hline Comunicación & 12 & 485,0000 & 40,4167 & A \\
\hline Mandos medios (supervisores) & 12 & 626,0000 & 52,1667 & A \\
\hline Clima laboral y moral de los empleados & 12 & 722,5000 & 60,2083 & $A$ \\
\hline $\begin{array}{l}\text { Sistema de ambiente, salud y seguridad: mejora de la } \\
\text { eficacia }\end{array}$ & 12 & 729,0000 & 60,7500 & $A$ \\
\hline Formación y desarrollo & 12 & 777,5000 & 64,7917 & A \\
\hline Conocimientos, actitudes y comportamientos & 12 & 856,0000 & 71,3333 & A \\
\hline Participación de los trabajadores & 12 & 858,0000 & 71,5000 & A \\
\hline Gerencia, cultura, liderazgo y prácticas gerenciales & 12 & 868,0000 & 72,3333 & A \\
\hline $\begin{array}{l}\text { Sistemas de trabajo, procesos y prácticas de los } \\
\text { empleados }\end{array}$ & 12 & 930,0000 & 77,5000 & A \\
\hline
\end{tabular}

Fuente: Autores.

Tabla 7

Procedimiento de comparación múltiple de Dunn: dimensiones cultura en seguridad de trabajadores operativos

\begin{tabular}{|c|c|c|c|c|c|}
\hline Dimensión & Frecuencia & Suma de los rangos & $\begin{array}{l}\text { Media de los } \\
\text { rangos }\end{array}$ & Grupc & \\
\hline Mandos medios (supervisores) & 70 & 20816,0000 & 297,3714 & A & \\
\hline Comunicación & 70 & 21306,5000 & 304,3786 & A & \\
\hline $\begin{array}{l}\text { Sistema de ambiente, salud y seguridad: mejora de la } \\
\text { eficacia }\end{array}$ & 70 & 22408,0000 & 320,1143 & A & \\
\hline Conocimientos, actitudes y comportamientos & 70 & 23431,0000 & 334,7286 & A & B \\
\hline El trabajo en equipo y colaboración & 70 & 23580,0000 & 336,8571 & A & B \\
\hline Participación de los trabajadores & 70 & 23920,5000 & 341,7214 & A & B \\
\hline Clima laboral y moral de los empleados & 70 & 24979,0000 & 356,8429 & A & B \\
\hline Formación y desarrollo & 70 & 27065,0000 & 386,6429 & A & B \\
\hline Gerencia, cultura, liderazgo y prácticas gerenciales & 70 & 27208,5000 & 388,6929 & A & B \\
\hline
\end{tabular}


Fuente: Autores.

\section{Liderazgo en seguridad}

El nivel promedio de liderazgo en seguridad percibido por los trabajadores encuestados resultó ser 4,063, con una desviación estándar de 0,786 , de forma que se ubica en un nivel medio-alto (Tabla 8).

Tabla 8

Nivel promedio de liderazgo en seguridad por nivel de cargo y empresa

\begin{tabular}{ccccc}
\hline Nivel de cargo & Empresa & Media & $\begin{array}{c}\text { Desviación } \\
\text { estándar }\end{array}$ & N \\
\hline Directivos y mandos & Empresa 1 & 4,290 & 0,593 & 2 \\
medios & Empresa 2 & 4,113 & 0,798 & 2 \\
& Empresa 3 & 4,016 & 0,420 & 8 \\
Operativos & Total & 4,078 & 0,462 & 12 \\
& Empresa 1 & 4,792 & 0,308 & 11 \\
& Empresa 2 & 3,889 & 0,563 & 11 \\
& Empresa 3 & 3,932 & 0,882 & 48 \\
Total & Total & 4,060 & 0,831 & 70 \\
& Empresa 1 & 4,715 & 0,379 & 13 \\
& Empresa 2 & 3,923 & 0,570 & 13 \\
& Empresa 3 & 3,944 & 0,830 & 56 \\
& Total & 4,063 & 0,786 & 82 \\
\hline
\end{tabular}

Fuente: Autores.

La prueba de normalidad de Kolmogorov-Smirnov, con el ajuste de Lilliefors, llevó a descartar el comportamiento normal de la variable liderazgo en seguridad ( $p$-valor $=0,008<0,05$ ), lo que hizo necesario recurrir al uso de pruebas no paramétricas para determinar diferencias significativas por nivel de cargo y por empresa.

La prueba U de Mann-Whitney llevó a descartar la existencia de diferencia significativa entre niveles de cargo en el nivel de liderazgo en seguridad ( $p$-valor $=$ $0,636>0,05$ ).

Por otra parte, el análisis de las dimensiones de la variable por nivel de cargo (Tabla 9) llevó a descartar diferencias significativas entre niveles de cargo.

Mediante la prueba de Kruskal-Wallis, se determinó que había diferencias significativas entre empresas en el nivel de liderazgo en seguridad ( $p$-valor $=6,81 \times 10-4<0,05$ ). Entre estas, el mayor nivel de cultura en seguridad es el de la empresa 1.
Tabla 9

Nivel promedio por dimensiones de la variable liderazgo en seguridad por nivel de cargo

\begin{tabular}{lccc}
\hline \multicolumn{1}{c}{ Dimensión } & Total & $\begin{array}{c}\text { Directivos y mandos } \\
\text { medios }\end{array}$ & Operativos \\
\hline Liderazgo directivos & 4,01 & 3,95 & 4,02 \\
Liderazgo mandos medios & 4,13 & 4,32 & 4,10 \\
Liderazgo responsables & 4,02 & 3,82 & 4,05 \\
Liderazgo en seguridad & $\mathbf{4 , 0 6}$ & $\mathbf{4 , 0 8}$ & $\mathbf{4 , 0 6}$ \\
\hline
\end{tabular}

Fuente: Autores.

De otra manera, el análisis de las dimensiones de la variable por empresa (Tabla 10) permitió encontrar diferencias significativas entre empresas en el nivel de todas las dimensiones. El mayor nivel fue el de la empresa 1 , en todas las dimensiones de la variable, y no hubo diferencias significativas entre las empresas 1 y 2 .

\section{Tabla 10}

Nivel promedio por dimensiones de la variable liderazgo en seguridad por empresa

\begin{tabular}{lcccc}
\hline \multicolumn{1}{c}{ Dimensión } & Total & Empresa 1 & Empresa 2 & Empresa 3 \\
\hline Liderazgo directivos & 4,01 & 4,73 & 3,87 & 3,87 \\
$\begin{array}{l}\text { Liderazgo mandos } \\
\text { medios }\end{array}$ & 4,13 & 4,82 & 4,07 & 3,99 \\
$\begin{array}{l}\text { Liderazgo } \\
\begin{array}{c}\text { responsables } \\
\text { Liderazgo en } \\
\text { seguridad }\end{array}\end{array}$ & $\mathbf{4 , 0 6}$ & $\mathbf{4 , 0 6}$ & 3,73 & 3,97 \\
\hline
\end{tabular}

Fuente: Autores.

Por otra parte, se descartó, mediante la prueba de Kruskal-Wallis, la existencia de diferencias significativas entre dimensiones en general ( $p$-valor $=0,560>0,05)$ y por nivel de cargo.

\section{Relación entre liderazgo en seguridad y cultura en seguridad}

A priori se observó una relación directa entre las variables liderazgo en seguridad y cultura en seguridad (Figura 1).

Se procedió a realizar la prueba de correlación de Pearson, para determinar relación entre el liderazgo en 
seguridad y la cultura en seguridad (Tabla 11). Como el p-valor estuvo muy por debajo de 0,05 , se confirmó una relación directa significativa entre el nivel de liderazgo en seguridad y el nivel de cultura en seguridad.

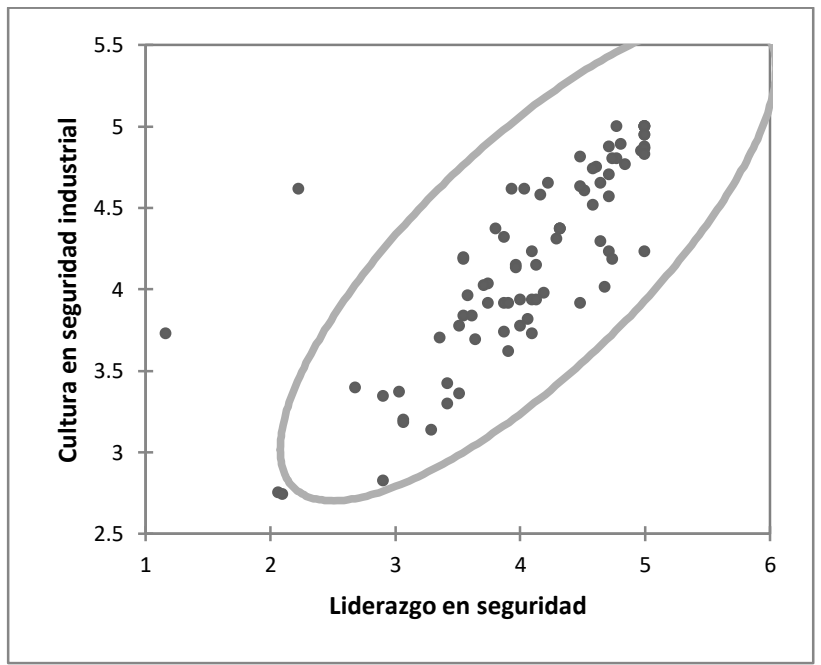

Figura 1. Liderazgo en seguridad vs. cultura en seguridad. Fuente: Autores.

\section{Tabla 11}

\section{Matriz de correlación variables de estudio}

\begin{tabular}{clcc}
\hline & & $\begin{array}{c}\text { Cultura } \\
\text { en } \\
\text { Seguridad }\end{array}$ & $\begin{array}{c}\text { Liderazgo } \\
\text { en } \\
\text { Seguridad }\end{array}$ \\
\hline $\begin{array}{c}\text { Cultura en } \\
\text { Seguridad }\end{array}$ & $\begin{array}{l}\text { Correlación } \\
\text { de Pearson } \\
\text { Sig. (bilateral) }\end{array}$ & 1 & \\
Liderazgo en \\
Seguridad & $\begin{array}{l}\text { Correlación } \\
\text { de Pearson }\end{array}$ & 0,786 & 1 \\
& $\begin{array}{l}\text { Sig. (bilateral) } \\
\text { < }\end{array}$ & & \\
\hline
\end{tabular}

Fuente: Autores.

Con el fin de verificar estos resultados, se hizo el análisis de regresión lineal (Tablas 12, 13 y 14). Para la aplicación del modelo, se verificó el cumplimiento del supuesto de independencia de errores, con el estadístico DurbinWatson, que tuvo un valor de 2,236 ; de modo que estuvo en el rango aceptable de 1,5 a 2,5.

El análisis de regresión lineal confirmó la relación directa entre las variables de estudio. De acuerdo con esto, el $61,4 \%$ de la variabilidad del nivel de cultura en seguridad se explica por el nivel de liderazgo en seguridad. Esto se resume en la siguiente ecuación:

$$
\hat{y}=1,755+0,598 X_{1}
$$

Donde:

$\hat{y}=$ Nivel de cultura en seguridad

$X_{1}=$ Nivel de liderazgo en seguridad

Tabla 12

Resumen del modelo

\begin{tabular}{ccccc}
\hline R & R cuadrado & $\begin{array}{c}\text { R cuadrado } \\
\text { corregida }\end{array}$ & $\begin{array}{c}\text { Error típ. } \\
\text { de la } \\
\text { estimación }\end{array}$ & $\begin{array}{c}\text { Durbin- } \\
\text { Watson }\end{array}$ \\
\hline 0,786 & 0,618 & 0,614 & 0,371 & 2,236 \\
\hline
\end{tabular}

Fuente: Autores.

Tabla 13

Análisis de varianza

\begin{tabular}{cccccc}
\hline Fuente & $\begin{array}{c}\text { Suma de } \\
\text { cuadrados }\end{array}$ & gl & $\begin{array}{c}\text { Media } \\
\text { cuadrática }\end{array}$ & F & Sig. \\
\hline Regresión & 17,804 & 1 & 17,804 & 129,655 & $\mathbf{0 , 0 0 0}$ \\
Residual & 10,985 & 80 & 0,137 & & \\
Total & 28,789 & 81 & & & \\
\hline
\end{tabular}

Fuente: Autores.

\section{Análisis de fiabilidad de la escala}

Antes de analizar el nivel promedio de la variable cultura en liderazgo y de sus dimensiones, se verificó la fiabilidad de la escala y las subescalas, con el coeficiente alfa de Cronbach. En la Tabla 15, se pueden observar los coeficientes alfa de Cronbach de las subescalas y escala.

Inicialmente los coeficientes alfa de Cronbach de las subescalas 1) sistemas de trabajo, procesos y prácticas de los empleados; 2) gerencia, cultura, liderazgo y prácticas gerenciales; 3) clima laboral y moral de los empleados; y 4) conocimientos, actitudes y comportamientos tuvieron un valor por debajo de 0,8 , por lo que fue necesario revisar los ítems con correlación ítem-total corregida por debajo de 0,350, y cuya eliminación contribuía a aumentar el coeficiente alfa de Cronbach. De esta manera, se eliminaron de cada subescala los ítems señalados en la Tabla 15.

Sin embargo, no fue posible elevar por encima de 0,8 el coeficiente de las subescalas 1) sistemas de trabajo, procesos y prácticas de los empleados y 2) clima laboral y moral de los empleados. No obstante, considerando que el coeficiente alfa de Cronbach de la escala resultó ser 0,981 , se validó que la escala como un todo tenía buena consistencia interna. 
Tabla 14

Parámetros del modelo

\begin{tabular}{ccccccc}
\hline Fuente & B & $\begin{array}{c}\text { Error } \\
\text { estándar }\end{array}$ & t & Sig. & \multicolumn{3}{c}{ Intervalo de confianza del 95\% para } \\
& & & & L. Inferior & L. Superior \\
\hline $\begin{array}{c}\text { (Constante) } \\
\text { Liderazgo en } \\
\text { Seguridad }\end{array}$ & 0,755 & 0,217 & 8,081 & $\mathbf{0 , 0 0 0}$ & 1,323 & 2,187 \\
\hline
\end{tabular}

Fuente: Autores.

\section{Tabla 15}

Coeficiente alfa de Cronbach subescalas / escala medición de variable cultura en seguridad

\begin{tabular}{ccc}
\hline Subescalas / Escala & Alfa de Cronbach & Ítems eliminados \\
\hline Sistemas de trabajo, procesos y prácticas de los empleados & 0,634 & $1,7,9$ \\
Gerencia, cultura, liderazgo y prácticas gerenciales & 0,861 & $14,16,21$ \\
Mandos medios (supervisores) & 0,951 & - \\
Formación y desarrollo & 0,911 & - \\
Comunicación & 0,914 & - \\
El trabajo en equipo y colaboración & 0,91 & - \\
Participación de los trabajadores & 0,868 & - \\
Clima laboral y moral de los empleados & 0,627 & - \\
Conocimientos, actitudes y comportamientos & 0,905 & $67,69,76,77,78,81,83$ \\
Sistema de ambiente, salud y seguridad: mejora de la eficacia & 0,916 & - \\
Cultura en seguridad industrial & 0,981 & - \\
\hline
\end{tabular}

Fuente: Autores.

Por otra parte, se verificó la fiabilidad de la escala y las subescalas de la variable liderazgo en seguridad, así como sus dimensiones con el coeficiente alfa de Cronbach. No fue necesario eliminar ninguno de los ítems (ver Tabla 16).

\section{Tabla 16}

Coeficiente alfa de Cronbach subescalas / escala de medición de variable liderazgo en seguridad

\begin{tabular}{|c|c|}
\hline Subescalas / escala & $\begin{array}{l}\text { Alfa de } \\
\text { Cronbach }\end{array}$ \\
\hline $\begin{array}{c}\text { Liderazgo en seguridad de } \\
\text { directivos }\end{array}$ & 0,949 \\
\hline $\begin{array}{l}\text { Liderazgo en seguridad de ma } \\
\text { medios y supervisores }\end{array}$ & 0,966 \\
\hline $\begin{array}{l}\text { Liderazgo en seguridad de } \\
\text { responsables de SG-SST }\end{array}$ & 0,940 \\
\hline Liderazgo en seguridad & 0,979 \\
\hline
\end{tabular}

Fuente: Autores.

\section{Capacitaciones realizadas y percepción sobre los riesgos críticos}

El número de promedio de capacitaciones a las que asistieron los trabajadores encuestados en el último año fue 4,07, con una desviación estándar bastante alta, de 3,64 capacitaciones. Esto oculta la baja participación en las capacitaciones. Un dato más diciente sería el siguiente: del $100 \%$ de los trabajadores encuestados, solo $41,46 \%$ asistió a 4 o más capacitaciones en el último año.

Del $100 \%$ de los trabajadores encuestados, 58,54\% recibió en el último año capacitación en manejo seguro de cargas; $54,88 \%$, en riesgo ergonómico; $48,78 \%$, en cómo actuar en caso de emergencia; $47,56 \%$, en uso adecuado de elementos de protección personal; 46,34 $\%$, en manejo de residuos; y $45,12 \%$, en estrés laboral.

Del $100 \%$ de los trabajadores encuestados, el 41,46 $\%$ tuvo accidentes laborales. El número promedio de accidentes laborales de los trabajadores encuestados 
que tuvieron accidentes laborales es 1,53 accidentes, con una desviación estándar de 0,707 accidentes.

En cuanto a las principales causas de ocurrencia de accidentes de trabajo, del $100 \%$ de los trabajadores encuestados, el 59,76\% identificó el exceso de confianza; el 35,37\%, la no utilización de elementos de protección personal; el 31,71\%, la distracción; y el 17,07 \%, el ritmo de trabajo muy acelerado.

Del $100 \%$ de los trabajadores encuestados, el 63,41\% consideró importante capacitarse en la identificación y manejo de los riesgos laborales propios de su labor.

Del $100 \%$ de los trabajadores encuestados, el 35,37\% manifestó que estaba al día o tenía bastante conocimiento de las normas de higiene y seguridad industrial aplicables a su labor.

Del $100 \%$ de los trabajadores encuestados, el 36,59\% manifestó realizar pausas activas dos o más veces al día.

En cuanto al uso de los elementos de protección personal, del $100 \%$ de los trabajadores encuestados, el $89,02 \%$ respondió utilizar guantes; el 60,98\%, gafas industriales; el 32,93 \%, casco; y el 31,71\%, tapabocas.

Con respecto a las dolencias sufridas en los últimos 3 meses, del $100 \%$ de los trabajadores encuestados, el $45,12 \%$ presenta dolencias por dolor de cuello y espalda; y el $28,05 \%$, dolor de cabeza. Sin embargo, el 36,59\% no presentó ninguna dolencia en los últimos 3 meses.

\section{Conclusiones}

Se confirmó la relación positiva y significativa entre el nivel de liderazgo en seguridad y el nivel de cultura en seguridad en los trabajadores de las empresas seleccionadas. El nivel de cultura en seguridad es mayor en la empresa 1, con respecto a las empresas 2 y 3 , y esto coincide con el mayor nivel de liderazgo en seguridad de la empresa 1. Esto concuerda con el hallazgo de MartínezOropesa y Cremades (2012) respecto a la relación entre cultura en seguridad y liderazgo en seguridad en trabajadores de empresas del sector industrial del Valle del Cauca.

Además, esto concuerda con lo planteado por Kouabenan, Ngueutsa y Mbaye (2015) respecto al papel clave de los directivos -y en especial los mandos medios- en la consolidación de la cultura de seguridad. De manera particular, el liderazgo ejercido en la ejecución del plan de capacitaciones contribuye en el aumento de la actitud favorable hacia la prevención de los riesgos laborales (Argüello-López, Uribe Bermúdez \& Valdivieso Guerrero, 2017).

Se verificó la consistencia interna de los cuestionarios utilizados para medir las variables. Fue necesario eliminar un número reducido de ítems de la escala para medir el nivel de cultura en seguridad, manteniendo invariable la escala, para medir el nivel de liderazgo en seguridad.

Este estudio permite plantear la utilización de las escalas como instrumentos válidos, tanto para medir el nivel de las variables cultura en seguridad y liderazgo en seguridad en un momento dado como para medir el efecto de las intervenciones que se realicen, con el objetivo de incrementar cada una de estas y de manera conjunta. La sistematización de los resultados obtenidos de la aplicación de las escalas en otras empresas permitirá validar sus características de fiabilidad.

Entre las limitaciones de este estudio se encuentran la toma de datos en un solo momento, lo que no permite determinar el efecto que tendría el nivel del liderazgo en seguridad en la efectividad de la implementación del SG-SST en cada una de las empresas. Lo anterior en la medida en que al momento de hacer la medición el nivel de implementación del SG-SST de la empresa 1 era menor con respecto a las demás empresas, lo que no corresponde con el mayor nivel de liderazgo en seguridad de esta empresa.

\section{Referencias}

Agencia Ejecutiva para la Salud y la Seguridad del Reino Unido. (2005). A review of safety culture and safety climate literature for the development of the safety culture inspection toolkit.

Argüello-López, G. M., Uribe Bermúdez, J. M., \& Valdivieso Guerrero, M. (2017). Relación entre capacitación y actitud hacia los riesgos laborales en el sector construcción del área metropolitana de Bucaramanga. I+D Revista de Investigaciones, 9(1), 14-26. https://doi.org/10.33304/revinv.v09n12017002

Cámara de Comercio de Bucaramanga. (2018a). ADN Sectorial Compite 360.

Cámara de Comercio de Bucaramanga. (2018b). Clasificación por tamaño de las empresas inscritas en Santander.

Cervantes, V. H. (2005). Interpretaciones del coeficiente Alpha de Cronbach. Avances En Medición, 3(1), 9-28.

Comisión de Seguridad y Salud del Reino Unido. (1993). ACSNI Study Group on Human Factors. 3rd Report: Organizing for Safety. Londres, Reino Unido: HSC.

FASECOLDA. (2018). Sistema de Consulta de Información 
en Riesgos Laborales - RL Datos Riesgos Laborales. Recuperado de https://fasecolda.com/index.php/ ramos/riesgos-laborales/documentos-de-interes/

Fernández-Muñiz, B., Montes-Peón, J. M., \& VázquezOrdás, C.J.(2004). Antecedentes del comportamiento del trabajador ante el riesgo laboral: Un modelo de cultura positiva hacia la seguridad. Revista de Psicología Del Trabajo y de Las Organizaciones, 21(3), 207-234.

Kapp, E. A. (2012). The influence of supervisor leadership practices and perceived group safety climate on employee safety performance. Safety Science, 50(4), 1119-1124. https://doi.org/10.1016/j.ssci.2011.11.011

Kouabenan, D. R., Ngueutsa, R., \& Mbaye, S. (2015). Safety climate, perceived risk, and involvement in safety management. Safety Science, 77, 72-79. https://doi. org/10.1016/j.ssci.2015.03.009

Martínez-Oropesa, C., \& Cremades, L. V. (2012). Liderazgo y cultura en seguridad: su influencia en los comportamientos de trabajo seguros de los trabajadores. Salud de Los Trabajadores, 20(2), 179-192.

Neal, A., Griffin, M. A., \& Hart, P. M. (2000). The impact of organizational climate on safety climate and individual behavior. Safety Science, 34(1-3), 99-109. https://doi.org/10.1016/S0925-7535(00)00008-4

Pidgeon, N. F. (1991). Safety culture and risk management in organizations. Journal of Cross-Cultural Psychology, 22(1), 129-140.

Pucci, F. (2007). Accidentes de trabajo y condiciones de riesgo en la industria de la construcción uruguaya. In La gestión del riesgo y las crisis: personas, culturas organizacionales e instituciones. (pp. 187-224). Buenos Aires, Argentina: FONCSI, Universidad de San Andrés y Editorial El Ateneo.

Puyal-Español, E. (2001). La conducta humana frente a los riesgos laborales: determinantes individuales y grupales. Acciones e Investigaciones Sociales, (12), 157-184.

Rimbau, E. (2013). Liderazgo y prevención de riesgos laborales. Seguridad y Salud En El Trabajo, 75, 10-17.

Vanegas, C., Jiménez, L., Cudris, L., \& Redondo, M. (2017). Ausentismo por enfermedad común en empleados de una central de energía -Antioquia. I+D Revista de Investigaciones, 9(1), 116-125. https://doi. org/10.33304/revinv.v09n1-2017011 